Evaluating internal validity in randomized controlled trials

\title{
Evaluating the degree of "unhappy randomization" in psychology: Are psychology experiments appropriately arranged to counter internal validity threats?
}

\author{
Chih-Long Yen ${ }^{\mathrm{a}}$ Chung-Ping Cheng ${ }^{\mathrm{b}^{*}}$ \\ ${ }^{a}$ Department of Counseling and I/O Psychology, Ming Chuan University, Taiwan. \\ ${ }^{\mathrm{b}}$ Department of Psychology, National Cheng Kung University, Taiwan.. \\ *C. P. Cheng and C. L. Yen contributed equally to this work.
}

\begin{abstract}
This study sought to evaluate whether the randomized controlled trials currently conducted in psychology are suitable for countering internal validity threats in terms of precluding alternative explanations. Experimental research primarily achieves the goal of drawing causal inferences through random assignment. As such, one of the threats to the internal validity of experiments is when random assignment fails to balance nuisance variables, thereby causing the treatment effect to be confounded by nuisance variables. The authors argue that the successful random assignment is largely decided by the sample size and the confounding effects that the researchers are willing to tolerate. In this study, one thousand and eighteen randomized controlled trials published in three leading psychology journals were investigated in order to estimate the probability that nuisance variables were balanced in these experiments. The results indicated that, even if researchers are willing to tolerate a medium effect size of confounding effects, there is still a high probability that over one-fifth $(22.99 \%)$ of the psychological experiments investigated could be confounded by nuisance variables, and the situation would be even worse if a stricter criterion was set. Some suggestions are proposed for enhancing the internal validity of future experiments.
\end{abstract}

Keywords: causal inference; experimental design; internal validity; Campbell's causal model 


\section{Evaluating internal validity in randomized controlled trials}

"Expectations [from random assignment] provide a strong foundation for causal inference.

Note these are expectations that hold exactly only in large samples ...In any single experiment, there is no guarantee that the mean pretest levels of the two treatment conditions will not differ on a specific covariate." (West \& Thoemmes ,2010, p.22)

\section{Introduction}

Internal validity has been seen as the "sine qua non" (Campbell \& Stanley, 1963, p. 175) of good experimental research because it is concerned with whether accurate inferences, regarding the relationship between independent and dependent variables, can be drawn from research results. In the related literature, various threats to internal validity and their remedies have been proposed (e.g., Campbell, 1957; Cook \& Campbell, 1979; Shadish et al., 2002). One of the major tasks for researchers is to identify plausible threats and then to include design elements that can potentially rule out these threats (West \& Thoemmes, 2010). Among them, random assignment is one of the primary methods employed by psychologists to ensure the internal validity of experiments. The proper random assignment of participants to experimental conditions results in the expectation that nuisance variables will be balanced across all the experimental groups, thus providing a foundation for the exclusion of plausible alternative explanations for the experimental results. However, can random assignment unconditionally guarantee balance across groups with regard to nuisance variables? For example, when conducting a two-condition experiment, if 400, 40, or even just 4 participants are randomly assigned to a treatment group and a control group, is the balance across the groups with regard to nuisance variables the same? The current study demonstrated that random assignment does not unconditionally guarantee balance across groups with regard to nuisance variables. The authors developed a method to identify the parameters needed for successful random assignment and to estimate the probability that a given psychology experiment 
has been appropriately arranged to counter internal validity threats. Specifically, the authors argue that a successful random assignment is largely decided by the sample size and the magnitude of confounding effects that the researchers are willing to tolerate. One thousand and eighteen randomized controlled trials published in three experimental psychology journals (namely, the Journal of Personality and Social Psychology, Psychological Science, and the Journal of Experimental Psychology: General) were investigated. More specifically, the probabilities of these experiments successfully ensuring the balance of nuisance variables across groups were evaluated.

\section{Internal Validity and Random Assignment}

The experimental method is seen as an effective method through which to infer causality, but the capacity to draw valid inferences from an experiment is not unconditional. One of the critical requirements for the validation of an experiment is internal validity (Cook \& Campbell, 1979), which was originally defined as the question, "did in fact the experimental stimulus make some significant difference in this specific instance?"(Campbell, 1957; p. 297). In other words, internal validity is concerned with whether or not the casual inference or inferences made based on an experiment are warranted (Shadish, Cook, \& Campbell, 2002). One of major threats to internal validity is the threat due to "selection," which refers to "systematic differences over conditions in respondent characteristics that also cause the observed effect" (Shadish, et al., 2002, p.55). Because the purpose of experiments is to eliminate possible alternative explanations for research results that are unrelated to the independent variable, when there are systematic differences in nuisance variables across experimental groups, even if the observed effect of the independent variable on a dependent variable is significant, it is impossible to be certain whether this association is due to the independent variable or due to nuisance variables. In practice, the primary means used by psychologists to exclude alternative explanations is the random assignment of participants to 
different conditions. Ideally, random assignment can balance all potential nuisance variables among the groups in an experiment. Assuming that $\varepsilon$ is the total effect that all nuisance variables have on $\mathrm{Y}$, regardless of how large $\varepsilon$ is, with random assignment, $\varepsilon$ would be equivalent for all levels of the independent variable $X$. Thus, the correlation between $X$ and $\varepsilon$ would be zero $\left(\mathrm{r}_{\mathrm{x} \varepsilon}=\right.$ $0)$. In an experiment, the observed effect of $\mathrm{X}$ on $\mathrm{Y}$ would then be confirmed and free of alternative explanations (because $r_{x \varepsilon}=0$ ). That is, it would not be possible to explain the differences in $\mathrm{Y}$ among groups by anything other than $\mathrm{X}$, so the threat of "selection" against internal validity would be eliminated.

\section{Random Assignment Only Provides a Probability and Not a Certainty}

Despite random assignment being an important tool in psychological experiments, it cannot guarantee success. This is because, for any unique experiment, achieving successful group balance is a matter of probability. Consider, for example,

“... a study with $N=20$ units, ten men and ten women, where the potential treatment and control outcomes are a priori thought to vary substantially by sex. Then, although a complete randomized design with $N_{t}=10$ [subscript " $t$ " denotes treatment group] would ensure that ten units get treated, there is the possibility that all ten of them are men (or women). In that case, average differences in the potential outcomes for active and control treatments could be due to sex differences rather than treatment effects." (Imbens \& Rubins, 2015, p51).

Indeed, the group balance achieved by random assignment in a specific experiment is probabilistic. As Cook and Campbell suggested that the "equivalence achieved by random assignment is probabilistic. Thus it is not inevitable that a correctly implemented randomization procedure will result in groups that do not differ" (Cook \& Campbell, 1979, p.341). In other words, we may sometimes have "unhappy randomization" (Shadish et al., 2002) in a specific 
experiment, such that it is possible that some nuisance variables highly imbalanced across groups, or equivalently, that there is a large $r_{x \varepsilon}$ in the experiment. Under unhappy randomization, the observed effect of $\mathrm{X}$ on $\mathrm{Y}$ is not free of alternative explanations, so the internal validity of an experiment is threatened (Shadish et al., 2002).

In most situations, it is hard to know whether or not a certain random assignment has been effective in balancing confounding effects because nuisance variables may be significant in number and may not all be measured or observed. What experimenters can do in a specific experiment is to increase the probability of group balance. Yet to what degree do current psychological experiments effectively balance nuisance variables and thereby preclude internal validity threats? Few past studies have investigated this issue and the consensus among researchers seems to be that random assignment is sufficient to ensure no interference from nuisance variables. However, as previously stated, random assignment only provides a probability and not a certainty. This implies that under certain circumstance, it may still be difficult to achieve group balance using random assignment. The goal of this study was to determine the parameters for successful random assignment and to use those parameters to assess the effectiveness of currently conducted psychology experiments in countering internal validity threats. The theoretical background of this study is explained below.

\section{Parameters Affecting Successful Random Assignment}

As mentioned previously, the purpose of random assignment is to make groups balanced in terms of the degrees to which they are related to any confounding effects, which means ensuring that the correlation between $X$ and $\varepsilon$ is zero $\left(r_{x \varepsilon}=0\right)$. However, because nuisance variables may be significant in number and because their effects may not all be measured or observed, the exact magnitude of $r_{x \varepsilon}$ in a specific experiment cannot be known. Nevertheless, the distribution of $r_{x \varepsilon}$ 
can be formulated. We can use this distribution to evaluate, under certain probability, if the $r_{\mathrm{x} \varepsilon}$ of a specific experiment is as small as the experimenters expect, thereby allowing experimenters to have confidence in the internal validity of their experiments.

To begin with the simplest case, assume an experiment with two conditions and that the experimenter wants to randomly assign $\mathrm{N}$ participants into an experimental group or control group. Let $\mathrm{X}$ be the independent variable and $\varepsilon$ be the cumulative effect of all nuisance variables on the dependent variable. Thus, the goal of random assignment is to balance the effects of nuisance variables in each group, that is, to make sure that there is no confounding variable related to independent variables. This is equivalent to letting the correlation between the independent variable and confounding effects be zero $\left(r_{x \varepsilon}=0\right)$. However, as previously stated, random assignment only provides a probability and not a certainty; thus, it is possible that a correctly implemented randomization procedure still results in imbalances between groups (Bowers, 2011; Cook \& Campbell, 1979; Imbens \& Rubins, 2015). That is, an $\mathrm{r}_{\mathrm{x} \varepsilon}$ not exactly equal to zero could happen even when random assignment is properly utilized. Moreover, what the nuisance variables are and how large the effect of the nuisance variables is are mostly unobserved by the researcher and, thus, the magnitude of the actual $r_{x \varepsilon}$ after random assignment in the experiment cannot be known. Nonetheless, the probability distribution of $r_{x \varepsilon}$ can be determined. Suppose a group of N participants is randomly assigned an infinite number of times, resulting in infinitely many $r_{x \varepsilon}$ values. As the assignment is random, the average of this distribution is 0 . The distribution of $r_{x \varepsilon}$ can be approximated by $t$ distribution with $\mathrm{N}-2$ degree of freedom and the standard deviation $\sigma_{\mathrm{r}}$ is $\frac{1}{\sqrt{\mathrm{N}-2}} .{ }^{1}$.

\footnotetext{
${ }^{1}$ Note: To the author's knowledge, there is no definitive distribution for the Pearson correlation in past literature. However, when the population correlation is $0, \frac{r_{X \varepsilon} / \sqrt{1-r_{X \varepsilon}^{2}}}{\frac{1}{\sqrt{\mathrm{N}-2}}}$ follows $t$ distribution with $N-2$ degree of freedom 
Although the $r_{x \varepsilon}$ distribution and its parameters can be determined, an actual experiment only involves the performance of one random assignment; therefore, for any given experiment, what the experimenter is concerned with is not the distribution of $r_{x \varepsilon}$ but the unique $r_{x \varepsilon}$ that is actually achieved in the particular experiment. This actual $r_{x \varepsilon}$ can be seen as one of the $r_{x \varepsilon} s$ in the $r_{x \varepsilon}$ distribution. It is impossible to know where this actual $r_{x \varepsilon}$ will lie in the distribution of $r_{x \varepsilon}$, but some conditions can be set to ensure a higher probability that it is within an acceptable range. For example, if we wish the actual $r_{x \varepsilon}$ to be within \pm .2 (i.e. we wish the magnitude by which the confounds interfere with the experiment to be less than .2) and the probability of the actual $r_{x \varepsilon}$ being within \pm .2 to be $95 \%$ (Figure 1 ), then, because the central value of the $r_{x \varepsilon}$ distribution is 0 , and because the $95 \%$ interval of $t$ distribution will be between $\pm \frac{.05}{2}, N-2$, we have

$$
\left|0 \pm t_{\frac{.05}{2}, N-2} \sigma_{\mathrm{r}}\right|<.2
$$

Where $\sigma_{\mathrm{r}}$ denotes the standardized error of the distribution of $r_{\mathrm{x} \varepsilon}$. Since $\sigma_{\mathrm{r}}=\frac{1}{\sqrt{\mathrm{N}-2}}$, it can be shown that:

$$
\left|0 \pm t_{\frac{.05}{2}, N-2} \frac{1}{\sqrt{\mathrm{N}-2}}\right|<.2
$$

Therefore, $\mathrm{N}$ should be larger than 100. That is, for an experiment with a sample size larger than 100 , the confounding effect, actual $r_{x \varepsilon}$, would have a $95 \%$ chance of being within \pm .2 .

From this it can be seen that in a one-factor two-level experiment, random assignment results are influenced by three parameters:

(Rahman, Macpherson, Rosemblatt \& Appelbaum, 1968). When $r_{X \varepsilon}$ is small, $\frac{r_{X \varepsilon}}{\sqrt{1-r_{X \varepsilon}^{2}}}$ approaches $r_{X \varepsilon}$; thus, the $r_{X \varepsilon}$ follows t distribution with N-2 degree of freedom and the standard deviation is $\frac{1}{\sqrt{\mathrm{N}-2}}$. While this deduction only stands when $r_{\mathrm{X} \varepsilon}$ is small, it is used only so that the readers can understand the logic behind the equations in this paper. The formula used thereafter is based on $\mathrm{F}$ distribution where $\mathrm{r}_{\mathrm{X} \varepsilon}$ is not restricted. 
(1) The upper bound of magnitude of a confounding effect which researchers are willing to tolerate. This is expressed as $r_{x \varepsilon . t o l}$, with the subscript "tol" indicating a confounding effect that a researcher is willing to "tolerate". Taking the previous calculation as an example, the largest confounding effect the researcher could tolerate is .2; therefore, $\mathrm{r}_{\mathrm{x} \varepsilon . \mathrm{tol}}=.2$.

(2) The probability of the actual confounding effect falling within the range of $\pm r_{x \varepsilon . t o l}$, which is expressed as $\mathrm{P}_{\text {within }}$ (where the subscript "within" indicates the probability that the confounding effect is "within" the range of $\left.\pm \mathrm{r}_{\mathrm{x} \varepsilon \text {.tol }}\right)$. Taking the previous calculation as an example, the probability the researcher expects that the actual $r_{x \varepsilon}$ falls within the range of $\pm r_{x \varepsilon . t o l}$ is $.95 ;$ therefore, $P_{\text {within }}=.95$.

(3) The sample size N.

The example above uses a one-factor two-level experiment to calculate the correlation between $\varepsilon$ and binary variable $\mathrm{X}$. This method could be extended to experiments with three or more conditions by considering multiple correlations between $\varepsilon$ and the experimental conditions. In a two-level experiment, the correlation coefficient $r_{x \varepsilon}$ is used to describe the relationship between the experimental groups and $\varepsilon$. In an experiment with three or more groups, the multiple correlation coefficient, $\mathrm{R}$, in a regression can be used to indicate $r_{x \varepsilon}$. This is because $\mathrm{k}$ groups of independent variable $\mathrm{X}$ can be expressed as k-1 dummy variables, a regression with $\varepsilon$ as a criterion variable and the k-1 dummy variables as predictors would indicate the relationship of $\varepsilon$ and $X$. The square root of the coefficient of determination $\left(R^{2}{ }_{x \varepsilon}\right)$ in this regression is the $R_{x \varepsilon}$ with which the experimenters are concerned. ${ }^{2}$ Because it is known that $\mathrm{R}^{2}$ follows the $\mathrm{F}$ distribution

\footnotetext{
${ }^{2}$ When $\mathrm{X}$ only has two groups, the multiple correlation is the Pearson correlation; therefore, the multiple correlation can also be used to cover the situation with only two groups. The use of dummy variables to find $R_{x \varepsilon}$ can be expanded to factorial experimental designs. For example, a $2 * 3$ experimental design includes six conditions. These six conditions can make $6-1=5$ dummy variables. The multiple correlation coefficients for the five dummy variables and $\varepsilon, R_{\left(D_{1}, D_{2}, D_{3}, \ldots, D_{5}\right), \varepsilon}$, is the extent of interference from the nuisance variables $\mathrm{R}_{\mathrm{x} \varepsilon}$.
} 
(Kirk, 1995, p.237). Thus, the F distribution can be used to infer the probability that that the actual confounding effects $\mathrm{R}_{\mathrm{x} \varepsilon}$ will be less than a certain criterion $\left(\mathrm{R}_{\mathrm{x} \varepsilon . \mathrm{tol}}\right)$. Hence, the parameters influencing the results of random assignment can be formulated as follows (the details of the derivation of this formula can be found in the appendix):

$$
\boldsymbol{P}_{\text {within }}\left(\boldsymbol{R}_{X \varepsilon}^{2}<\boldsymbol{R}_{X \varepsilon . t o l}^{2}\right)=p\left(\boldsymbol{F}_{k-1, N-k}<\frac{\frac{\boldsymbol{R}_{X \varepsilon . t o l}^{2}}{k-1}}{\frac{\boldsymbol{1}-\boldsymbol{R}_{X \varepsilon . t o l}^{2}}{N-k}}\right)
$$

where $\mathrm{R}_{\mathrm{x} \varepsilon \text {.tol }}$ is the upper limit of the confounding effects that the researchers are willing to tolerate, $\mathrm{P}_{\text {within }}$ is the probability of the magnitude of the actual confounding effects $\mathrm{R}_{\mathrm{x} \varepsilon}$ being less than $\mathrm{R}_{\mathrm{x \varepsilon .tol}}, \mathrm{k}$ is the number of conditions, and $\mathrm{N}$ is the sample size. For example, if an experimenter is conducting a 5 conditions experiment with 100 participants and sets $R_{x \varepsilon . t o l}=.1$, then that indicates that the experimenter wishes for the confounding effects to be less than small effect (that is, $k=5, N=100, \mathrm{R}_{\mathrm{x} \varepsilon . \mathrm{tol}}=.1$ in this case). In addition, because the result of random assignment is probabilistic, the experimenter has to decide the desired probability of the actual confounding effects falling within the tolerable range. If the experimenter wishes for there to be a $95 \%$ chance that the actual confounding effects will fall within the tolerable range, the experimenter will set $\mathrm{P}_{\text {within }}=.95$.

In summary, according to Equation 1, the result of random assignment is decided by four parameters: The number of experimental conditions $(\mathrm{k})$, the confounding effects that the researchers are willing to tolerate $\left(\mathrm{R}_{\mathrm{x} \varepsilon . \mathrm{tol}}\right)$, the probability of the actual confounding effect being less than $\mathrm{R}_{\mathrm{x} \varepsilon \text {.tol }}\left(\mathrm{P}_{\text {within }}\right)$, and the sample size. Accordingly, given $\mathrm{R}_{\mathrm{x} \varepsilon \text {.tol }}$ and $\mathrm{k}$, we can calculate $\mathrm{P}_{\text {within }}$ of a given experiment in terms of its sample size. That is, by using the given sample size, we can evaluate if the result of a random assignment achieves the experimenter's expectation of ruling out the interference from confounding effects in the experiment. This study used Equation 
1 to evaluate the probability of success of random assignment in psychological experiments. To test the validity of this equation, a simulation using empirical data was conducted; the results

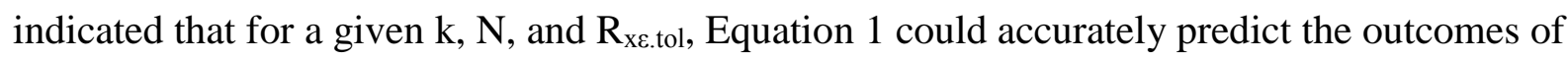
random assignment (see supplementary material).

\section{An Example Illustrating the Meaning of Equation}

Here, an example is used to further explain the meaning of Equation 1, and to compare it with the traditional power analysis approach. In recent years, psychology has focused on replication in the hopes of helping psychological research to better meet scientific standards (Open Science Collaboration, 2015; Pashler \& Wagenmakers, 2012). The results of a number of psychological studies have been found to be irreproducible. One focus of considerable debate has been studies regarding embodied cognition. Zhong and Liljenquist (2006) used four randomized controlled trials to explain that one's moral purity induces a need for cleanliness. The priming of immorality increased the mental accessibility of cleansing-related concepts and a greater desire for cleansing products. However, later researchers found that these results could not be replicated (see Simonsohn, 2015). Equation 1 was used to calculate the probability of success of random assignment for Zhong and Liljenquist's (2006) four experiments; the results are shown in Table

1.

The values in the gray area of Table 1 are the probability of the confounding effects being within the tolerable range $\left(\mathrm{P}_{\text {within }}\right)$ as calculated using the number of experimental conditions $(\mathrm{k})$ and the actual sample size (N) found in Zhong and Liljenquist's (2006) study given an acceptable

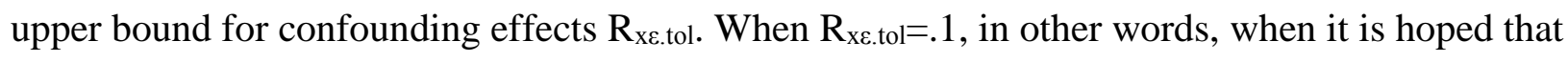
the interference from confounding effects is no more than a small effect, then the probability of the four experiments reaching this is between .37 and .55 (the first gray column underlined in 
Table 1). This means that there is an over $45 \%$ chance that the confounding effects would exceed .1. When a more lax upper limit is given, $\mathrm{R}_{\mathrm{x} \varepsilon . \mathrm{tol}}=.2$, then the probability of the four experiment reaching this is between .67 and .87 (the second gray column underlined in Table 1). This means that despite allowing a greater confounding effect, there is an over $13 \%$ chance that the confounding effects would exceed .2 .

Moreover, for a $95 \%$ probability that the confounding effects are controlled within the acceptable range $\left(\mathrm{P}_{\text {within }} \geqq .95\right)$, in other words, only a 5\% chance (adopting the same criterion as the traditional type I error rate, $\alpha=.05$ ) that the confounding effects exceed the tolerable limit, then only $\mathrm{R}_{\mathrm{x} \text {.tol}}=.4$ will allow $\mathrm{P}_{\text {withins }}$ to be close to .95 given the circumstances of Zhong and Liljenquist's (2006) four experiments (the fourth underlined column on the right in Table 1); however, $R_{x \varepsilon . t o l}=0.4$ is a standard difficult to accept. Therefore, Zhong and Liljenquist's (2006) experiments could, according to Equation 1, have had a large probability of being heavily influenced by nuisance variables. This may be one of the reasons that their results have been irreproducible.

In summary, Equation 1 developed in this study found that the success of random assignment is affected by four parameters: The confounding effect researchers are willing to tolerate $\left(\mathrm{R}_{\mathrm{x} \varepsilon . \mathrm{tol}}\right)$, the probability of the actual $\mathrm{R}_{\mathrm{x} \varepsilon}$ falling within the range of $\mathrm{R}_{\mathrm{x} \varepsilon . \mathrm{tol}}\left(\mathrm{P}_{\text {within }}\right)$, the sample size $(\mathrm{N})$, and the number of experimental conditions $(\mathrm{k})$. When given any three of these four parameters, the fourth parameter can be found. Equation 1 was then used to evaluate whether or not the psychology experiments currently being conducted are appropriately arranged to eliminate confounding effects and thereby ensure their own internal validity.

\section{Method and Materials}


Studies in three high impact experimental psychology journals, namely, the Journal of Personality and Social Psychology, Psychological Science, and the Journal of Experimental Psychology-General, were investigated. All the papers published from 2011 to 2015 in these three journals were reviewed. Those papers containing the words 'random', 'assign', 'between', or 'condition' were then further selected. A preliminary test (using 28 papers) revealed that these four words can effectively and rapidly help in judging whether or not a given paper utilized a random assignment design (with an accuracy rate of $100 \%$ in the preliminary test). Selected papers were then checked by the first author. If the experimental design of a target paper was confirmed to be a random assignment design, one experiment was randomly selected from the paper as research material. That is, our research materials comprised almost all the papers using complete random assignment designs which were published from 2011 to 2015 in the three target journals (with at least one experiment in each paper). According to the above procedure, a total of 1018 experiment were selected. For each experiment, the number of experimental conditions (k) and the sample size $(\mathrm{N})$ were coded. The sample sizes of these 1018 experiments ranged from 4 to 6548 , with a $M d=95$.

According to Equation 1, given the number of experimental conditions (k), and the magnitude of the confounding effect the researchers were willing to tolerate $\left(\mathrm{R}_{\mathrm{x} \varepsilon \mathrm{tol}}\right)$, we can use the sample size of each experiment to calculate the probability of the actual confounding effect falling within the range of $\mathrm{R}_{\mathbf{x} \text {.tol }}$ (i.e., $\mathrm{P}_{\text {within }}$ ). As the analysis results from the three journals were similar, they were combined as one analysis to more clearly express the results (readers interested in the results for different journals, please see supplementary document, Table S2). The results are shown in Table 2. The values in Table 2 indicate the number (and ratio) of experiments which met the given criteria. 
As can be seen in Table 2, most randomized controlled trials in psychology seem to be to be inappropriately arranged in terms of eliminating confounding effects. At $\mathrm{P}_{\text {within }}=.95$, that is, with only a 5\% chance (adopting the same criterion as the traditional type I error rate, $\alpha=.05$ ) that the confounding effects will exceed the acceptable limit, and the tolerable limit $\left(\mathrm{R}_{\mathrm{x \varepsilon .tol}}\right)$ is set at .1 (i.e., a small effect size), only 33 of these 1018 (3.24\%) psychological experiments meet these criteria (data in the first row and the third column highlighted in gray in Table 2). In other words, $96.57 \%$ of the experiments cannot meet the criteria given a small effect size of confounding effects. Even after relaxing this to $\mathrm{R}_{\mathrm{x} \varepsilon . \mathrm{tol}}=.2$, only 315 of the 1018 (30.94\%) experiments meet these criteria (data in the second row and the third column highlighted in gray in Table 2). In other words, close to $70 \%$ of experiments do not meet the $\mathrm{R}_{\mathrm{x} \varepsilon . \mathrm{tol}}=.2$ and $\mathrm{P}_{\text {within }}=.95$ requirements. If $\mathrm{R}_{\mathrm{x} \varepsilon . \mathrm{tol}}=.3$ is acceptable (which is equivalent to a medium effect size), 784 of the $1018(77.01 \%)$ experiments meet these criteria (data in the third row and the third column highlighted in gray in Table 2); this indicates that over one fifth of experiments $(1-77.01 \%=22.99 \%)$ cannot satisfy the requirements for random assignment even given a medium effect size of confounding effects. Therefore, given a traditional type I error rate $\left(\mathrm{P}_{\text {within }}=.95\right)$, unless the confounding effects are allowed to reach $.4, .5$, or higher, the majority of psychological experiments cannot satisfy the requirements for success of random assignment. In other words, the confounding effects in these experiments may not be small and the internal validity of the experiments may be threatened by nuisance variables.

At $\mathrm{P}_{\text {within }}=.80$, that is, with a $80 \%$ chance that the confounding effects will be within the acceptable limits, and $\mathrm{R}_{\mathrm{x} \varepsilon . \mathrm{tol}}$ is set at .1, only 104 of the $1018(10.22 \%)$ experiments meet these criteria (data in the first row and the second underlined column in Table 2). In other words, close to $90 \%$ of the experiments cannot meet the criteria given a small effect size of confounding effects. If the $\mathrm{R}_{\mathrm{x} \varepsilon \text {.tol }}$ is relaxed to $.2,708(69.55 \%)$ of the experiments meet these criteria (data in 


\section{Evaluating internal validity in randomized controlled trials}

the second row and the second underlined column in Table 2). Therefore, even at $\mathrm{P}_{\text {within }}=.80$, unless the confounding effects are allowed at $\mathrm{R}_{\mathrm{x} \varepsilon . \mathrm{tol}}=.3$ or higher, there would still be close to one third of experiments $(1-69.55 \%=30.45 \%)$ unable to satisfy the requirements for success of random assignment. In summary, the above analysis indicates that many current randomized controlled trials in psychology seem to be inappropriate for their internal validities.

\section{Discussion}

Internal validity is crucial for experiments. It has been seen as a "basic minimum" (Campbell, (1957, p.297) of experiments. A major requirement for internal validity is to make nuisance variables balance across groups thereby precluded the confounding effects from experiments. However, according to the analysis, the appropriateness of most psychological experiments in precluding confounding effects may be questionable in terms of the four parameters mentioned in the study. That is, current randomized controlled trials in psychology utilize random assignment as a major means against internal validity threats, but the interference of nuisance variables might be greater than researchers expect. Therefore, the internal validity of these experiments may be debatable.

Randomized controlled trials have long been considered the gold standard for psychological experiments. Most psychologists believe that there is no need to consider covariates because they believe that random assignment ensures that groups are balanced in terms of nuisance variables effects, therefore excluding the possible confounding effects from experiments. However, the method presented here shows that random assignment cannot unconditionally make nuisance variables balance across groups. There are two main causal model approaches, Campbell's causal model and Rubin's causal model, which have been discussed thoroughly in previous studies (Rubin, 2010; Shadish, 2010). Campbell’ s causal model focuses more on experimental design, 
which largely relies on random assignment to eliminate threats to internal validity, while Rubin' s causal model focuses more on data analysis, which suggests including possible covariates to form matched groups or to get better estimations of the experimental effects. The authors suggest that researchers synthesize these two models. When the sample size is large and the number of experimental conditions is small, random assignment is indeed an elegant way to attain group balance in terms of the effects of any nuisance variables. However, when the sample size is not large enough and the number of experimental conditions is large, simple random assignment may be insufficient for preventing threats to internal validity. Therefore, even for randomized controlled trials, covariates, such as a pretest or any known potential nuisance variable, should be included in the experiments for statistical control when the sample size is small and the experimental design is complex.

According to Equation 1, the probability of group imbalance is determined not only by the sample size but also by the number of experimental conditions. The greater the number of experimental conditions, the larger the sample size needed to balance nuisance variables. However, in the analysis of 1018 experiments, the correlation between the number of conditions and sample size was only .24 (.081 if influential cases are deleted). This suggested that researchers seem not to take the number of experimental conditions into account seriously when deciding the sample sizes in their experiments. Although the relationships among sample size, number of conditions, and magnitude of confounding effects are a matter a probability, from the perspective of Campbell's causal model, it is better to have a design that rules out threats to internal validity in the beginning than it is to rely upon statistical analysis to determine whether a threat is plausible after an experiment has been conducted (Shadish, 2010). In other words, Campbell's causal model stresses the importance of experimental design over analysis. Just as Light, Singer, and Willett (1990) stated, “you can’t fix by analysis what you bungled by design” 
(p. viii). This is exactly what we claim in this paper. Although random assignment has been seen as an effective way of diminishing selection bias, it is not unconditional. Experimenters should try their best to achieve the success of random assignment at the start of an experiment rather than expect that nuisance variable balance will naturally occur simply through randomization. We thus suggest that psychologists should take their experimental designs, sample sizes, and the confounding effects they are willing to tolerate into account in a serious way before conducting experiments.

One of parameters for deciding the probability of appropriately balancing nuisance variables across groups is the confounding effect that the researchers are willing to tolerate. It should be noted, however, that while we stated this parameter as if a researcher can decide the magnitude of tolerable confounding effect on his/her own, the magnitude of this criterion should, in fact, be defined based on the consensus among members of the scientific community, like the magnitude setting for the nominal type I error rate. Because the goal of an experimental design is to exclude the influences of nuisance variables and to allow for valid inferences of causality, a small effect size for $\mathrm{R}_{\mathrm{x} \varepsilon \text {.tol }}$ (i.e, $\mathrm{R}_{\mathrm{x} \varepsilon . \mathrm{tol}}=.10$; Cohen, 1988) could be a reasonable requirement worth incorporating. The purpose of a given experiment should also be considered. In our opinion, larger magnitudes of tolerable confounding effects might be appropriate for exploratory studies, but smaller magnitudes should be considered for confirmatory studies. In summary, the criterion of tolerable confounding effects should not be unconditional or subjective; rather, a researcher should provide arguments for choosing the magnitude for such criterion.

In summary, although random assignment is widely viewed as the "gold standard" of experimental design, the present paper suggests that experimental psychologists have largely ignored the prerequisites needed for random assignment, and have thus caused their research to have a high risk of poor internal validity, which may in turn threaten the validity of current 
knowledge in the field of psychology. The authors wish this paper could remind psychologists to rethink about the relationship of internal validity and experimental design.

\section{References}

Bakker, M., van Dijk, A., \& Wicherts, J. M. (2012). The rules of the game called psychological science. Perspectives on Psychological Science, 7(6), 543-554.

Bowers, J. (2011). Making effects manifest in randomized experiments. In J. N. Druckman, D. P. Green, J. H. Kuklinski, A. Lupia \& A. Arbor (Eds.), Cambridge handbook of experimental political science (pp. 459-480). New York: Cambridge University Press.

Campbell, D. T. (1957). Factors relevant to the validity of experiments in social settings. Psychological Bulletin, 54, 297-312.

Campbell, D. T., \& Stanley, J. C. (1963). Experimental and quasi-experimental designs for research on teaching. In N. L. Gage (Ed.), Handbook of research on teaching (pp. 171-246). Chicago: Rand McNally.

Cohen, J. (1988). Statistical power analysis for the behavioral sciences. Hillsdale, N.J.: L. Erlbaum.

Cook, T. D., \& Campbell, D. T. (1979). Quasi-experimentation: Design and analysis for field settings. Boston: Houghton Mifflin.

Imbens, G. W., \& Rubin, D. B. (2015). Causal inference in statistics, social, and biomedical sciences. NY: Cambridge University Press.

Kirk, R. E. (1995). Experimental design: Procedures for the behavioral sciences (3rd ed.). Pacific Grove, CA: Brooks/Cole.

Light, R. J., Singer, J. D., \& Willett, J. B. (1990). By design: Planning research in higher education. Cambridge, MA: Harvard University Press.

Lindsay, D. S. (2015). Replication in Psychological Science. Psychological Science, 26(12), 1-6. 
Open Science Collaboration. (2015). Estimating the reproducibility of psychological science. Science, 349(6251), 943.

Pashler, H., \& Wagenmakers, E. J. (2012). Editors' Introduction to the Special Section on Replicability in Psychological Science A Crisis of Confidence? Perspectives on Psychological Science, 7(6), 528-530.

Rahman, N. A., Macpherson, A. S., Rosemblatt, K. A., \& Appelbaum, N. P. (1968). A course in theoretical statistics: for sixth forms, technical colleges, colleges of education, universities. London: Griffin.

Revelle, W. (2015). psych: Procedures for personality and psychological research (R Package Version 1.5.8) [Computer software]. Retrieved from http://cran.rproject.org/web/packages/psych/index.html

Rubin, D. B. (2010). Reflections stimulated by the comments of Shadish (2010) and West and Thoemmes (2010). Psychological Methods, 15(1), 38-46.

Shadish, W. R. (2010). Campbell and Rubin: A primer and comparison of their approaches to causal inference in field settings. Psychological Methods, 15(1), 3-17. doi: 10.1037/a0015916

Shadish, W. R., Cook, T. D., \& Campbell, D. T. (2002). Experimental and quasi-experimental designs for generalized causal inference. Boston: Houghton Mifflin.

Simonsohn, U. (2015). Small Telescopes Detectability and the Evaluation of Replication Results. Psychological Science, 26(5), 559-569.

Steiner, P. M., Cook, T. D., Shadish, W. R., \& Clark, M. H. (2010). The importance of covariate selection in controlling for selection bias in observational studies. Psychological Methods, $15(3), 250$.

Zhong, C., \& Liljenquist, K. (2006). Washing away your sins: Threatened morality and physical cleansing. Science, 313, 1451-1452. 
Evaluating internal validity in randomized controlled trials

Table 1

The probability of success of random assignment for Zhong and Liljenquist's (2006) four experiments

\begin{tabular}{|c|c|c|c|c|c|c|c|}
\hline \multirow[t]{2}{*}{$\begin{array}{l}\text { Experiments } \\
\text { in } Z \text { \& L }\end{array}$} & \multirow{2}{*}{$\begin{array}{l}\text { Number of } \\
\text { conditions } \\
\text { (k) }\end{array}$} & \multirow{2}{*}{$\begin{array}{c}\text { Sample } \\
\text { size } \\
(\mathrm{N})\end{array}$} & \multicolumn{5}{|c|}{$\begin{array}{l}\text { Probability of successful random assignment } \\
\qquad\left(\mathrm{P}_{\text {within }}\right)\end{array}$} \\
\hline & & & $\mathrm{R}_{\mathrm{x} \varepsilon \mathrm{tol}}=.1$ & $\mathrm{R}_{\mathrm{x} \varepsilon \mathrm{tol}}=.2$ & $\mathrm{R}_{\mathrm{x} \text {.tol }}=.3$ & $\mathrm{R}_{\mathrm{x} \varepsilon \mathrm{tol}}=.4$ & $\mathrm{R}_{\mathrm{x \varepsilon} . \mathrm{tol}}=.5$ \\
\hline Exp. 1 & 2 & 60 & .55 & .87 & .98 & $\underline{1.00}$ & 1.00 \\
\hline Exp. 2 & 2 & 26 & .37 & .67 & .86 & .96 & .99 \\
\hline Exp. 3 & 2 & 32 & .41 & .73 & .90 & .98 & 1.00 \\
\hline Exp. 4 & 2 & 45 & .49 & .81 & .95 & .99 & 1.00 \\
\hline
\end{tabular}


Evaluating internal validity in randomized controlled trials

Table 2

Number and ratio of experiments of which actual $R_{x \varepsilon}$ fell within the tolerable range

\begin{tabular}{|c|c|c|c|}
\hline & & $\begin{array}{l}\text { ber of exper } \\
{(\text { ratio })^{\mathrm{a}}}\end{array}$ & \\
\hline & $\mathrm{P}_{\text {within }}=.50$ & $\mathrm{P}_{\text {within }}=.80$ & $\mathrm{P}_{\text {within }}=.95$ \\
\hline \multirow[t]{2}{*}{$\mathrm{R}_{\mathrm{x} \varepsilon \text {.tol }}=.1$} & 516 & $\underline{104}$ & 33 \\
\hline & $(50.69 \%)$ & $(10.22 \%)$ & $(3.24 \%)$ \\
\hline \multirow[t]{2}{*}{$\mathrm{R}_{\mathrm{x} \varepsilon . \mathrm{tol}}=.2$} & 961 & $\underline{708}$ & 315 \\
\hline & $(94.40 \%)$ & $(69.55 \%)$ & $(30.94 \%)$ \\
\hline \multirow[t]{2}{*}{$\mathrm{R}_{\mathrm{x} \varepsilon . \mathrm{tol}}=.3$} & 1015 & $\underline{988}$ & 784 \\
\hline & $(99.71 \%)$ & $(97.05 \%)$ & $(77.01 \%)$ \\
\hline \multirow[t]{2}{*}{$\mathrm{R}_{\mathrm{x} \varepsilon . \mathrm{tol}}=.4$} & 1017 & $\underline{1013}$ & 978 \\
\hline & $(99.90 \%)$ & $(99.51 \%)$ & $(96.07 \%)$ \\
\hline \multirow[t]{2}{*}{$\mathrm{R}_{\mathrm{x} \varepsilon . \mathrm{tol}}=.5$} & 1018 & $\underline{1017}$ & 1013 \\
\hline & $(100.00 \%)$ & $(99.90 \%)$ & (99.51\%) \\
\hline
\end{tabular}

${ }^{\text {a }}$ Denominator is 1018 experiments. 


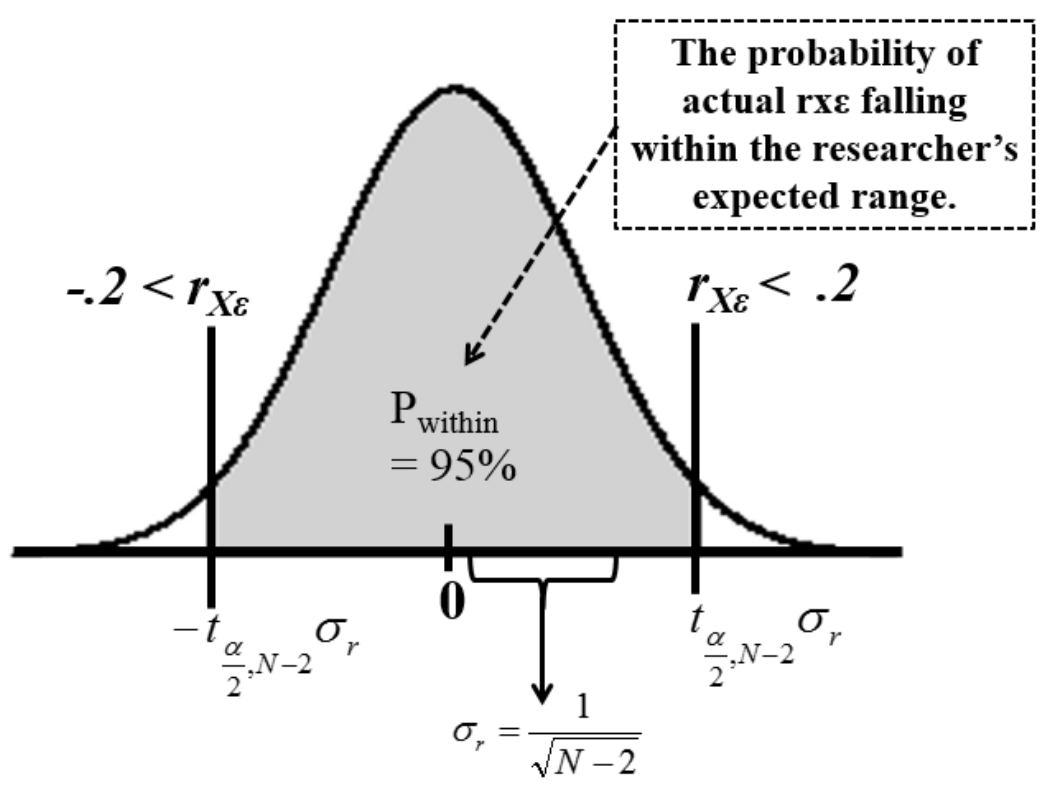

Figure 1 Sampling distribution of $\mathrm{r}_{\mathrm{x} \varepsilon}$ 


\section{Appendix}

In this appendix, we demonstrate that the probability of the actual confounding effect falling within in a specified range in a k-group experiment can be calculated through the F distribution.

As we have mentioned in the main text of this paper, the degree to which the confounding effect or effects interfere with a specific experiment can be expressed by the magnitude of $R_{x \varepsilon}$. Suppose the independent variable $\mathrm{X}$ includes $\mathrm{k}$ groups, then $\mathrm{X}$ can be expressed as $\mathrm{k}-1$ dummy variables. To indicate the relationship of $\varepsilon$ and $\mathrm{X}$, we can conduct a regression with $\varepsilon$ as a criterion variable and the $\mathrm{k}-1$ dummy variables as predictors. The square root of the $\mathrm{R}_{\mathrm{x} \varepsilon}^{2}$ in this regression is the $\mathrm{R}_{\mathrm{x} \varepsilon}$ with which the experimenters are concerned. When the experimenters set a prespecified criterion for the confounding effect which they are willing to tolerate (i.e, $R_{x \varepsilon . t o l}$ ), the probability of the actual confounding effect falling within the prespecified range is expressed as $\boldsymbol{P}_{\text {within }}\left(\boldsymbol{R}_{X \varepsilon}^{2}<\boldsymbol{R}_{X \varepsilon . t o l}^{2}\right)$. Since $\frac{\frac{\boldsymbol{R}_{X \varepsilon}^{2}}{\boldsymbol{k - 1}}}{\frac{1-\boldsymbol{R}_{X \varepsilon}^{2}}{N-\boldsymbol{k}}}$ follows $\boldsymbol{F}_{k-1, N-k}(\mathrm{Kirk}, 1995, \mathrm{p} .237)$, the probability of

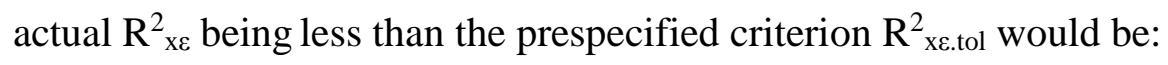

$$
\boldsymbol{P}_{\text {within }}\left(\boldsymbol{R}_{X \varepsilon}^{2}<\boldsymbol{R}_{X \varepsilon . t o l}^{2}\right)=\boldsymbol{P}_{\text {within }}\left(\frac{\frac{\boldsymbol{R}_{X \varepsilon}^{2}}{k-1}}{\frac{1-\boldsymbol{R}_{X \varepsilon}^{2}}{N-k}}<\frac{\frac{\boldsymbol{R}_{X \varepsilon . t o l}^{2}}{k-1}}{\frac{1-R_{X \varepsilon . t o l}^{2}}{N-k}}\right)=P_{w i t h i n}\left(F_{k-1, N-k}<\frac{\frac{\boldsymbol{R}_{X \varepsilon . t o l}^{2}}{k-1}}{\frac{1-R_{X \varepsilon . t o l}^{2}}{N-k}}\right)
$$

